\title{
Rotigotine is safe and efficacious in Atypical Parkinsonism Syndromes induced by both $\alpha$-synucleinopathy and tauopathy
}

This article was published in the following Dove Press journal:

Neuropsychiatric Disease and Treatment

5 June 2014

Number of times this article has been viewed

\author{
Davide Vito Moretti \\ Giuliano Binetti \\ Orazio Zanetti \\ Giovanni Battista Frisoni \\ IRCCS San Giovanni di Dio \\ Fatebenefratelli, Brescia, Italy
}

Correspondence: Davide Vito Moretti IRCCS San Giovanni di Dio

Fatebenefratelli, Brescia, Italy

$\mathrm{Tel}+39030350 \quad$ I597

$\mathrm{Fax}+390303533513$

Email davide.moretti@afar.it

\begin{abstract}
Transdermal rotigotine (RTG) is a non-ergot dopamine agonist (D3 $>$ D2 $>$ D1), and is indicated for use in early and advanced Parkinson's disease (PD). RTG patch has many potential advantages due to the immediacy of onset of the therapeutic effect. Of note, intestinal absorption is not necessary and drug delivery is constant, thereby avoiding drug peaks and helping patient compliance. In turn, transdermal RTG seems a suitable candidate in the treatment of atypical Parkinsonian disorders (APS).

Fifty-one subjects with a diagnosis of APS were treated with transdermal RTG. The diagnoses were: Parkinson's disease with dementia, multiple system atrophy Parkinsonian type, multiple system atrophy cerebellar type, progressive supranuclear palsy, corticobasal degeneration, Lewy body dementia, and frontotemporal dementia with Parkinsonism. Patients were evaluated by the Unified Parkinson's Disease Rating Scale (UPDRS; part III), Neuropsychiatric Inventory (NPI), and mini-mental state examination (MMSE) and all adverse events (AEs) were recorded.

Patients treated with RTG showed an overall decrease of UPDRS III scores without increasing behavioral disturbances. Main AEs were hypotension, nausea, vomiting, drowsiness, tachycardia, and dystonia. On the whole, 15 patients were affected by AEs and seven patients suspended RTG treatment due to AEs.

The results show that transdermal RTG is effective with a good tolerability profile. RTG patch could be a good therapeutic tool in patients with APS.
\end{abstract}

Keywords: transdermal dopamine agonist, open label study, Parkinson plus

\section{Introduction}

Atypical Parkinsonian disorders (APS) are a group of diseases that share extrapyramidal features with Parkinson's disease (PD), but with a globally different clinical picture and different neuropathological substrates. The most recognized APS are: progressive supranuclear palsy (PSP), corticobasal degeneration (CBD), multiple system atrophy (MSA), Parkinson's disease with dementia (PDD), and Lewy body dementia (LBD). PSP, CBD, MSA, PDD, and LBD are distinct pathological entities. ${ }^{1-5}$ The pathophysiology of APS is still unknown and the therapeutic choices are very limited. For example, the major limitation to the use of dopaminergic agents in APS is the high risk of inducing psychotic adverse events (AEs) and behavioral disturbances. ${ }^{6,7}$

Recently, a novel agent has been approved for PD: transdermal rotigotine (RTG). RTG is a non-ergolinic dopamine agonist (DA) administered via a transdermal patch that delivers the drug over a 24 -hour period. ${ }^{8}$ The RTG transdermal patch is available in various dosages (ie, $2-8 \mathrm{mg}$ ).

The application of the RTG transdermal patch provides predictable release and absorption of RTG. A lot of recent well-designed and large clinical trials have 
demonstrated that RTG produces significant improvements in the Unified Parkinson's Disease Rating Scale (UPDRS) summed motor and activities of daily living (ADL) scores as compared to placebo. ${ }^{9-14}$ Moreover, transdermal RTG has been demonstrated ${ }^{9}$ to improve motor functioning, sleep disturbances, nighttime motor symptoms, pain and functioning, and depressive symptoms. On the whole, RTG was welltolerated across the trials. ${ }^{10-15}$ Thus, the RTG transdermal patch offers a useful therapeutic tool for the treatment of motor and non-motor symptoms in extrapyramidal disorders like APS.

In most of the clinical trials carried out so far, transdermal RTG has been administered only in patients with idiopathic PD. In the present study, RTG was administered to 51 patients with APS, showing overall good performance on motor symptoms without inducing behavioral or psychiatric disorders usually seen when dopaminergic agonists are used in patients with cognitive disorders associated with extrapyramidal features.

\section{Methods}

\section{Subjects}

Fifty-one subjects admitted to the memory clinic of the National Institute for Research and Cure of Neurodegenerative Disorders Fatebenefratelli (FBF) in Brescia, Italy were diagnosed as having APS. Transdermal RTG was administered to all 51 patients. Subjects were evaluated by boardcertified neurologists and movement disorder specialists who work in the Memory Clinic and Movement Disorders Center. All experimental protocols were approved by the local ethics committee. Informed consent was obtained from all participants or their caregivers, according to the Code of Ethics of the World Medical Association (Declaration of Helsinki). Table 1 shows sociodemographic data for the whole study group.

\section{Diagnostic criteria}

In our subjects, diagnoses were as follows: PDD (nine patients), MSA-Parkinsonism (MSA-P; eleven), MSA-cerebellar type (MSA-C; four), PSP (seven), CBD (ten), LBD (nine), and frontotemporal dementia with parkinsonism (FTD-P; one). In this study, the subjects affected by PSP were all the PSP-Parkinsonism (PSP-P) type. Moreover, all diagnoses

Table I Sociodemographical data of the whole study group

\begin{tabular}{llllll}
\hline Subjects & MMSE & UPDRS/56 & NPI/I44 & Age & Education \\
\hline 5 I & 19.9 & 35.2 & 57.6 & 70.4 & 6.4 \\
\hline
\end{tabular}

Note: Age and education are expressed in years.

Abbreviations: MMSE, mini-mental state examination; NPI, Neuropsychiatric Inventory; UPDRS, Unified Parkinson's Disease Rating Scale.
Table 2 Number of subjects and clinical characteristics at baseline, T6, TI2, and TI8 follow-up

\begin{tabular}{lllll}
\hline & Baseline & T6 & TI2 & T I8 \\
\hline Subjects & 51 & $45(6)$ & $30(1)$ & $21(0)$ \\
MMSE & $19.9( \pm 1.2)$ & $18.9( \pm 2.3)$ & $17.2( \pm 0.4)$ & $14.1( \pm 1.8)$ \\
UPDRS & $35.2( \pm 2.2)$ & $28.1( \pm 3.1)$ & $26.4( \pm 2.9)$ & $25.5( \pm 4.3)$ \\
NPI & $57.6( \pm 2.4)$ & $52.1( \pm 3.09)$ & $51.5( \pm 1.5)$ & $38.8( \pm 0.9)$ \\
RTG dosage & ND & $3.2( \pm 0.2)$ & $4.2( \pm 0.5)$ & $5.1( \pm 1.1)$ \\
\hline
\end{tabular}

Note: The number of subjects that dropped out are shown within the parentheses. Abbreviations: MMSE, mini-mental state examination; ND, no data; NPI, Neuropsychiatric Inventory; RTG, rotigotine; UPDRS, Unified Parkinson's Disease Rating Scale; T, time.

were performed exclusively on clinical evaluation. Subjects were titrated with transdermal RTG to the minimal effective dosage (Table 2).

Diagnostic criteria of APS were inferred from literature guidelines determined by the most influential previous studies and largely accepted clinical evidence. ${ }^{16-23}$ Briefly, the main clinical features of the classical PSP phenotype are postural instability, early falls, early cognitive decline with frontal symptoms, and abnormalities of vertical gaze; this is known as Richardson's syndrome (RS). PSP has been subdivided into two main clinical and pathological phenotypes: classical RS and PSP-P. PSP-P differs from the classical form because of an asymmetric onset, tremor, and a mild-to-moderate early therapeutic response to levodopa.,24 As regards to CBD, the classical clinical picture consists of asymmetric Parkinsonism and cortical signs (eg, apraxia, cortical sensory loss, and alien limb). Dystonia and myoclonus can be part of the syndrome. It is usually defined as corticobasal syndrome (CBS) when there is not a neuropathological confirmation. MSA is helpfully recognized by the presence of Parkinsonism, autonomic dysfunction, and mixed cerebellar and pyramidal signs. MSA is, in turn subdivided into MSA-P or MSA-C type based on the predominant phenotype at onset. In this study, patients who developed dementia more than 1 year after the onset of clinically definite PD were included. The diagnosis of clinically definite PD was based on previously published criteria: the presence of at least two of the three cardinal signs of akinesia, rigidity, and resting tremor. Other criteria required were unilateral onset and the development of postural instability associated with significant responsiveness to a dopaminergic agent. ${ }^{25-28}$ According to the clinical guidelines of LBD diagnosis, patients with cognitive impairment developed less than 1 year from the appearance of Parkinsonian symptoms, repeated falls, hallucinations at disease onset, or symptom fluctuations, and vivid dreams were included in this group. ${ }^{29,30}$ The main concomitant pathologies of the patients in the study sample were: hypertension, 
hypercholesterolemia, hypertensive cardiopathy, type 2 diabetes mellitus, and carotid atheroma. As a consequence, patients were taking antihypertensive, antiplatelet, and antidiabetic drugs. The patients were encouraged to maintain the concomitant treatments as stably as possible. Patients who presented psychosis were not admitted to the study. No other anti-Parkinsonian agents were taken by the patients before or during the study. Memory, visuospatial skills, and language were the most affected cognitive domains.

\section{Clinical evaluation}

All patients underwent complete physical and neurological examinations. All patients underwent an electroencephalographic (EEG) and magnetic resonance imaging (MRI). Only 27 patients underwent single photon emission computed tomography dopamine transporter scan (SPECT-daT scan). MRI is useful for excluding vascular Parkinsonism, whereas SPECT shows diffuse and more symmetric degeneration of nigrostriatal pathways, as well as excluding idiopathic PD, which usually has a clearly asymmetric impairment. Patients were evaluated with the Unified Parkinson's disease rating scale (UPDRS) part III (out of a total of 56 points), ${ }^{31} \mathrm{Neu}-$ ropsychiatric Inventory (NPI), ${ }^{32}$ and the mini-mental state examination (MMSE) ${ }^{33}$ at baseline and after 6, 12, 18 months of follow-up (T0, T6, T12, T18). All AEs were recorded.

\section{Statistical analysis}

The Kolmogorov-Smirnov test was applied to check the distribution of the sample population. The result was $P=0.76$, thus confirming the normal distribution of the sample. Oneway analysis of variance (ANOVA), with Newman-Keuls post-hoc correction, was performed to test the changes in the MMSE, UPDRS-III, and NPI, at all time points (T0, T6, T12, T18). Post-hoc test analysis was performed only when the main analysis had a significant result $(P<0.05)$ in order to explore which of the single comparisons were statistically significance. Age and education were considered to be covariates in the main ANOVA.

\section{Results}

At baseline, the mean UPDRS-III score was 35.2/56, the mean NPI score was 57.6/144, and the mean MMSE score was $19.9 / 30$. Forty-five of $51(88.2 \%)$ patients completed 6 months of treatment with RTG (mean dose $=3.24 \mathrm{mg}$ / 24 hours): the mean UPDRS-III score was 28.1/56, the mean NPI score was 52.1/144, and the mean MMSE score was $18.9 / 30$. Six patients dropped out due to AEs. Thirty of $51(58.8 \%)$ patients completed 12 months of treatment with
RTG (mean dose $=4.2 \mathrm{mg} / 24$ hours): the mean UPDRS-III score was 26.4/56, the mean NPI score was 51.5/144, and the mean MMSE score was 17.2/30. One more patient dropped out due to AEs. Twenty-one of 51 (41.1\%) patients completed 18 months of treatment with RTG (mean dose $=5.1 \mathrm{mg}$ / 24 hours): the mean UPDRS-III score was 25.5/56, the mean NPI score was 38.8/144, and the mean MMSE score was 14.1/30. No patients dropped out at the 18-month follow-up. Reported AEs were: hypotension (13 patients), nausea (ten), vomiting (four), drowsiness (four), tachycardia (two), dystonia (three patients with anterocollis, all treated with concomitant L-3,4-dihydroxyphenylalanine). On the whole, 15 patients were affected by AEs (29.4\%) and seven patients suspended RTG treatment due to AEs (13.7\%; vomiting, tachycardia, and sleepiness). No heart congestion failure was detected among our patients.

The subscore of the NPI scale regarding delusions and hallucinations was examined. At baseline, the mean scores were $2 / 12$ for delusion and 1.5/12 for hallucinations. These scores remained invariate at $\mathrm{T} 6$ and $\mathrm{T} 12$, and did not change statistically significantly at T18 (1.8/12 and 1.3/12, respectively).

Figure 1 shows the ANOVA results for UPDRS. A significant decrease of the score $(P<0.00005)$ was found. After post-hoc analysis, all comparisons with the baseline were statistically significant $(P<0.0002)$.

Figure 2 shows the ANOVA results for NPI. A significant decrease of the score $(P<0.003)$ was found. After post-hoc analysis, all comparisons with the T18 follow-up were statistically significant $(P<0.004)$.

Figure 3 shows the ANOVA results for MMSE. A significant decrease of the score $(P<0.0004)$ was found. After post-hoc analysis, all comparisons with the T18 follow-up were statistically significant $(P<0.02)$.

\section{Discussion}

\section{Current treatment options in APS}

Recently there has been growing attention in trying to identify a possible therapy for sporadic APS conditions. The monoamine oxidase-B inhibitor rasagiline ( $1 \mathrm{mg} /$ day) was administered for 48 weeks in 174 patients with possible or probable MSA-P type, in a randomized, placebocontrolled clinical trial. No significant differences were found in the total Unified Multiple System Atrophy Rating Scale (UMSARS) score between the treatment and placebo groups. ${ }^{34}$ Infusions of $0.4 \mathrm{~g} / \mathrm{kg}$ intravenous immunoglobulin for 6 months were administered in a single-arm, singlecenter, open-label pilot trial in seven patients. In this trial, 
UPDRS-III across 18 months follow-up

$\mathrm{F}(3,141)=12.40 ; P<0.0000$

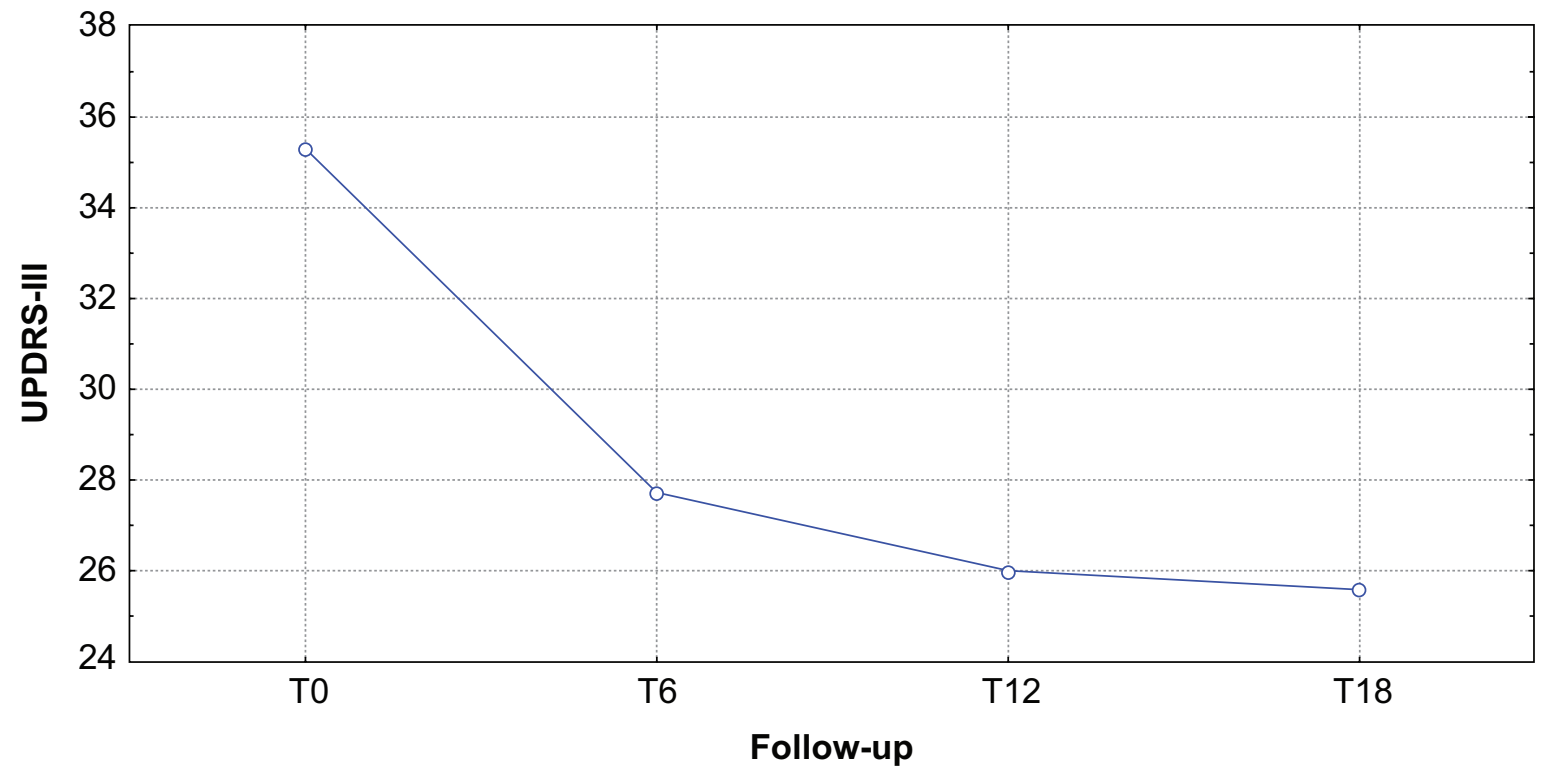

Figure I ANOVA results for UPDRS score.

Abbreviations: ANOVA, analysis of variance; UPDRS, Unified Parkinson's Disease Rating Scale; T, time.

a significant improvement was found in UMSARS part I (ADL) and II (motor functions) ${ }^{35}$ Thirty-three patients with probable MSA-C type were treated with 30-50 intra-arterial or intravenous injections of autologous mesenchymal stem cells (MSCs) and compared with placebo. The results demonstrated that the MSC group had an increase in total and part II UMSARS scores from baseline after a 360-day follow-up period. ${ }^{36}$

\section{NPI across 18 months follow-up}

$\mathrm{F}(3,141)=3.14 ; P<0.0273$

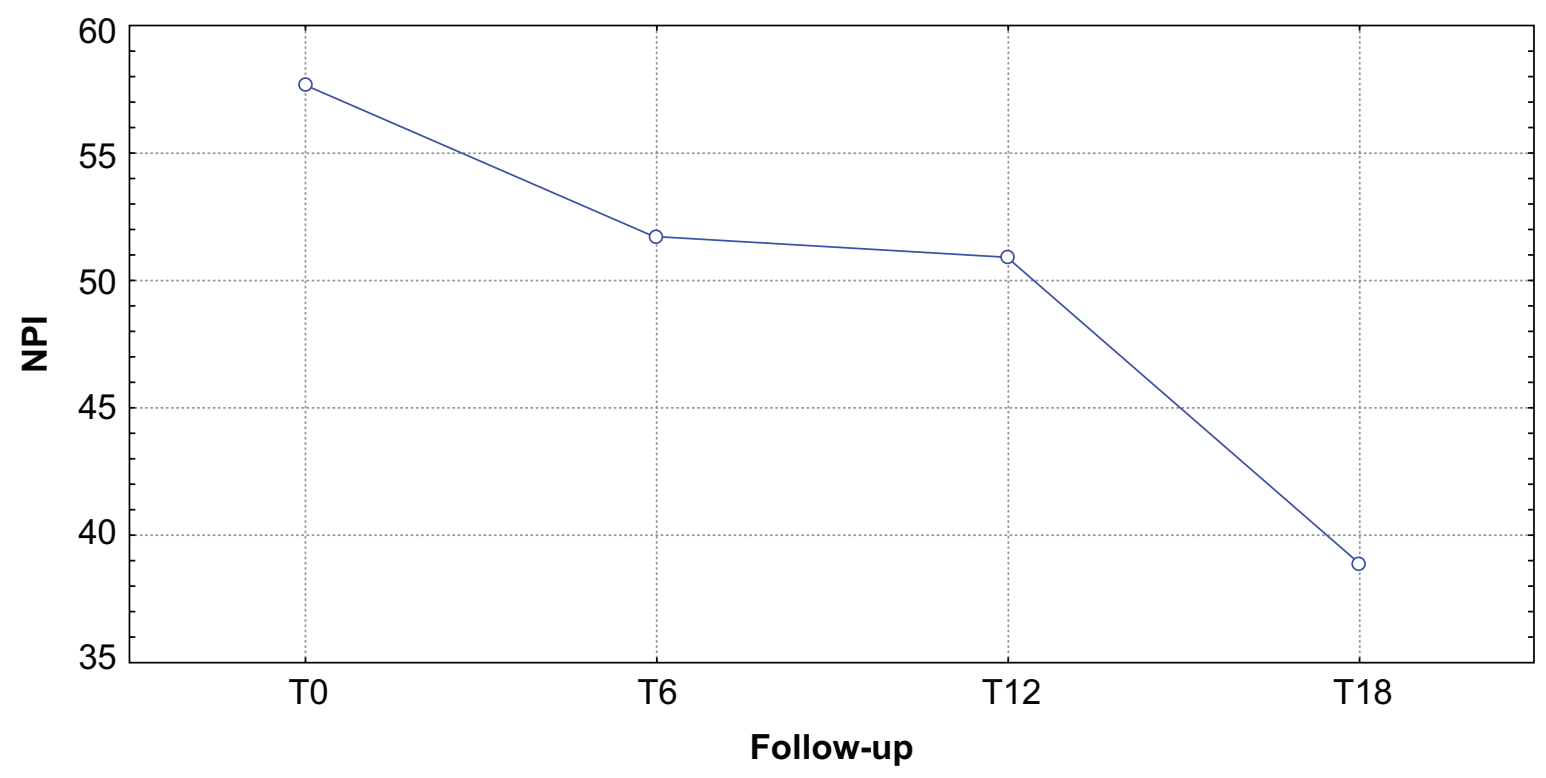

Figure 2 ANOVA results for NPI score.

Abbreviations: ANOVA, analysis of variance; NPI, Neuropsychiatric Inventory; T, time. 
MMSE across 18 months follow-up

$F(3,141)=6.40 ; P<0.0004$

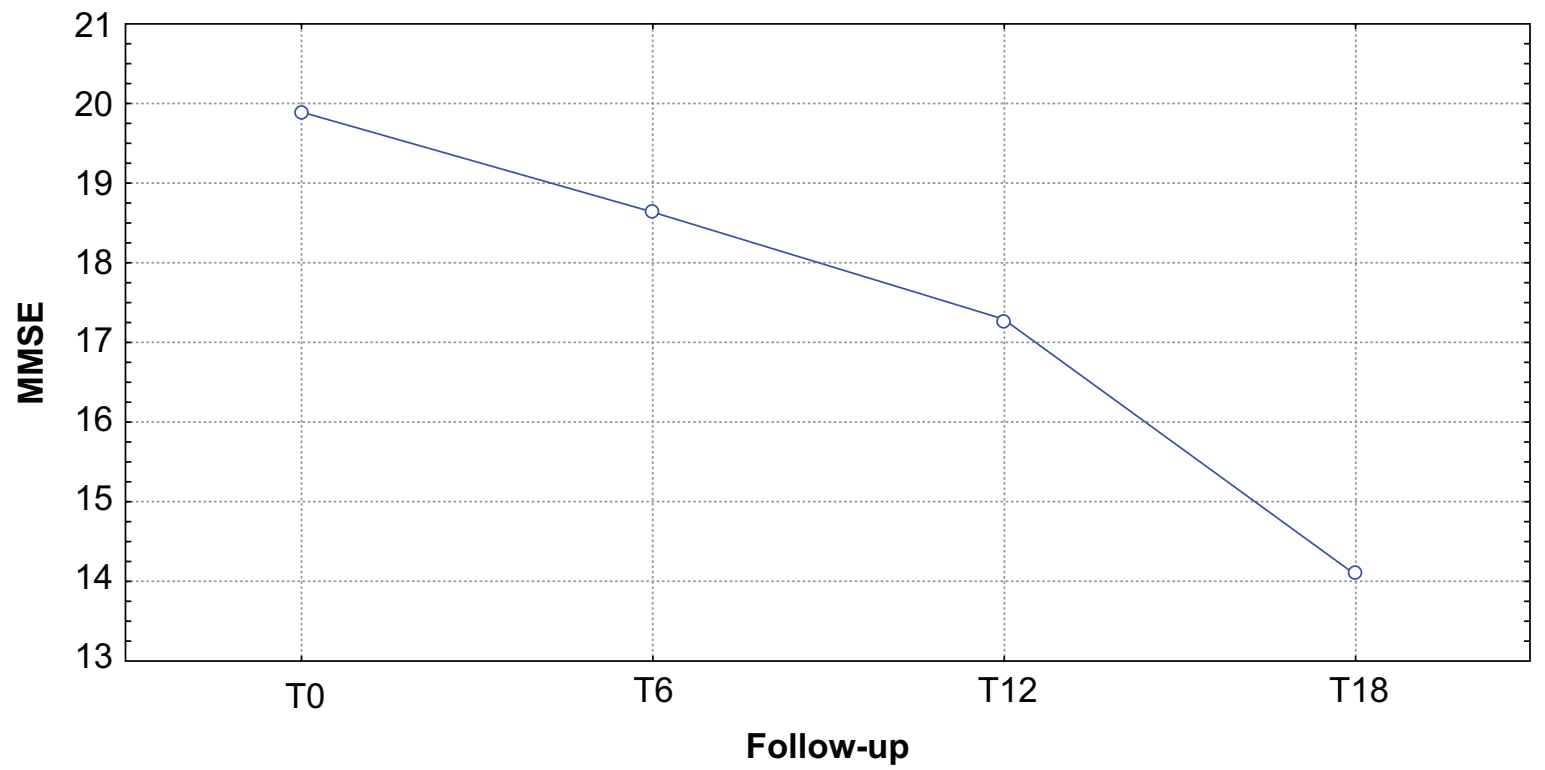

Figure 3 ANOVA results for MMSE score.

Abbreviations: ANOVA, analysis of variance; MMSE, mini-mental state examination; T, time.

As regards to PSP, 313 participants were treated with $30 \mathrm{mg}$ davunetide or placebo twice daily for 52 weeks in a randomized, double-blind, placebo-controlled trial. No significant effects were found on the Progressive Supranuclear Palsy Rating Scale (PSPRS) and the Schwab and England Activities of Daily Living (SEAD; see on http//www.fiercebiotech.com/press-release/allon-announcespsp-clinical-trials-results, press release by Allon Therapeutics on December 18, 2012). Inhibitors of glycogen synthase kinase-3 (GSK-3), were also administered in patients with APS. One hundred and forty-two patients with PSP were treated orally with tideglusib (600 $\mathrm{mg}$ or $800 \mathrm{mg}$ per day), in a Phase II, double-blind, placebo-controlled trial or placebo for 1 year. There were no significant differences between the high dose and low dose, as well as between the drug versus the placebo groups in the outcome measures..$^{37,38}$ Finally, an open-label pilot trial of lithium, also an inhibitor of GSK-3, in 17 patients both with PSP and CBD (ClinicalTrials.gov identifier NCT00703677) was stopped prematurely because of the presence of severe AEs.

\section{Transdermal RTG as a treatment option for APS}

Transdermal RTG seems to be effective and well-tolerated in patients with APS. Our results show significant improvement in UDPRS-III scores, maintained along the course of the 18 months' follow-up. Moreover, only seven patients dropped out, out of the 15 patients affected by AEs. These results confirm previous evidence obtained in patients with idiopathic PD showing positive effect on motor control. ${ }^{10-15}$ A plausible explanation of the results is that transdermal RTG provides continuous dopaminergic stimulation, reducing the unwanted motor effects of immediate release dopaminergic therapies. Our results also show a reduction of NPI scores, which became significant at the last follow-up evaluation (T18), confirming previous evidence in PD studies. According to these studies, RTG patients have absolute reductions in off-time that are similar to those reported in comparable studies with the oral DAs pramipexole or ropinirole. The clinical relevance of the observed improvement in off-time and on motor functions is further supported by a significant improvement in the quality of life, as seen in the mobility, ADL, and emotional well-being domains of the Parkinson's Disease Questionnaire (PDQ)-39. The AE profiles of both active treatments in this study were similar. Typical dopaminergic side effects were seen, and both drugs were well-tolerated. There were small differences in the frequency of dopaminergic side effects with RTG and pramipexole: there were more reports of nausea in patients receiving RTG than in those on pramipexole; by contrast, patients on pramipexole reported hallucinations, dyskinesias, and dizziness more frequently than did patients receiving RTG. 
Although efficacy and systemic tolerability were similar for transdermal RTG and oral pramipexole and ropinirole, transdermal drug delivery could still offer several important benefits for the treatment of patients with Parkinsonian symptoms. One of these is related to dosing via once daily patch administration, which could potentially enhance compliance, particularly in patients on regimens with several oral drugs (eg, in patients with motor fluctuations, where the daily tablet intake is often above ten). Nonadherence to prescribed medication has been identified as a major problem in APS patients, both with respect to missed doses and mistiming of scheduled doses, which has an obvious effect on the quality of control of motor oscillations. Another potential advantage of transdermal drug delivery in PD is related to the lack of drug-food interactions with such delivery systems. Such a delivery system could be advantageous in clinical settings with patients experiencing impaired swallowing, which is common in patients with APS. ${ }^{39,40}$ Of note, it has been demonstrated that the administration of RTG in patients with poor morning motor control reduces the morning motor symptoms, sleep disturbances, and other non-motor symptoms. ${ }^{12-14}$ Patients with extrapyramidal symptoms are particularly prone to the side effects of anti-Parkinsonian dopaminergic therapy, such as symptoms of depression and anxiety, hallucinations, delusions (with prevalent paranoid symptoms), agitation, and delirium. ${ }^{41,42}$ Furthermore, treatment with DAs is the main risk factor for developing impulse control disorders (ICDs). The most common ICDs reported with other DAs like ropinirole, pramipexole, bromocriptine, as well as the ergot-DA, are pathological gambling, hypersexuality, and compulsive shopping and eating. ${ }^{6-8}$ Our study did not find behavioral or psychiatric AEs nor ICDs. This could be explained by the particular pharmacological action of RTG, which is different from other dopaminergic agents. At first, RTG has its highest affinity for D3 receptors, ${ }^{43}$ whereas other dopaminergic agents share their main action on both D2/D3 receptors. D3 is localized in the caudate-putamen region, with particular concentrations in the ventral striatum. This peculiar position seems to be of a crucial importance in modulating the affective state. ${ }^{43}$ Another important difference with other DAs is that transdermal RTG also has high activity at non-dopaminergic receptors, in particular the $\alpha 2 \mathrm{~B}$-adrenergic receptors and the serotonin 5-HT1A receptors, which could positively modulate mood and behavior. The duration of the therapeutic effect on other motor and behavioral symptoms for up to 18 months of follow-up could suggest a neuroprotective effect. Although previously seen in animal models and in vitro studies, ${ }^{43,44}$ this aspect needs to be cautiously assessed further. During our study, none of the patients suffered from congestive heart failure. In this aspect, the low affinity of RTG for 5-hydroxytryptamine receptor $2 \mathrm{~B}$ (5-HT2B) may be of clinical importance. All other ergolinic DAs provoking cardiac valvular damage are full or partial 5-HT2B receptor agonists. ${ }^{45}$

The MMSE score shows that patients were highly cognitively impaired, on average, at the beginning of the study. Our results show no positive effect on cognitive status. However, further studies with less initially cognitively compromised patients grouped for single pathologies will better clarify this issue.

\section{Conclusion}

RTG appears to be a suitable therapy in elderly patients as it has a good tolerability profile, improves patients' compliance, and helps management of fragile patients. D3 receptor activation in the caudate-putamen region by RTG compensates for hypodopaminergic function in pathways in these areas and is probably responsible for the efficacy of this drug.

\section{Disclosure}

The authors report no conflicts of interest in this work.

\section{References}

1. Litvan I, Bhatia KP, Burn DJ, et al; Movement Disorders Society Scientific Issues Committee. Movement Disorders Society Scientific Issues Committee report: SIC Task Force appraisal of clinical diagnostic criteria for Parkinsonian disorders. Mov Disord. 2003;18(5):467-486.

2. Williams DR, de Silva R, Paviour DC, et al. Characteristics of two distinct clinical phenotypes in pathologically proven progressive supranuclear palsy: Richardson's syndrome and PSP-parkinsonism. Brain. 2005; 128(Pt 6):1247-1258.

3. Gilman S, Wenning GK, Low PA, et al. Second consensus statement on the diagnosis of multiple system atrophy. Neurology. 2008;71(9): 670-676.

4. Wenning GK, Geser F, Krismer F, et al; European Multiple System Atrophy Study Group. The natural history of multiple system atrophy: a prospective European cohort study. Lancet Neurol. 2013;12(3): 264-274.

5. Stamelou M, Quinn NP, Bhatia K. “Atypical” atypical parkinsonism: new genetic conditions presenting with features of progressive supranuclear palsy, corticobasal degeneration, or multiple system atrophy-a diagnostic guide. Mov Disord. 2013;28(9):1184-1199.

6. Antonini A, Cilia R. Behavioural adverse effects of dopaminergic treatments in Parkinson's disease: incidence, neurobiological basis, management and prevention. Drug Saf. 2009;32(6):475-488.

7. Vilas D, Pont-Sunyer C, Tolosa E. Impulse control disorders in Parkinson's disease. Parkinsonism Relat Disord. 2012;18 Suppl 1:S80-S84.

8. Schnitzler A, Leffers KW, Häck HJ. High compliance with rotigotine transdermal patch in the treatment of idiopathic Parkinson's disease. Parkinsonism Relat Disord. 2010;16(18):513-516.

9. Trenkwalder C, Kies B, Rudzinska M, et al; Recover Study Group. Rotigotine effects on early morning motor function and sleep in Parkinson's disease: a double-blind, randomized, placebo-controlled study (RECOVER). Mov Disord. 2011;26(1):90-99.

10. Baldwin CM, Keating GM. Rotigotine transdermal patch: a review of its use in the management of Parkinson's disease. CNS Drugs. 2007;21(12): 1039-1055. 
11. Poewe WH, Rascol O, Quinn N, et al; SP 515 Investigators. Efficacy of pramipexole and transdermal rotigotine in advanced Parkinson's disease: a double-blind, double-dummy, randomised controlled trial. Lancet Neurol. 2007;6(6):513-520.

12. Sanford M, Scott LJ. Rotigotine transdermal patch: a review of its use in the treatment of Parkinson's disease. CNS Drugs. 2011;25(8):699-719.

13. LeWitt PA, Boroojerdi B, Surmann E, Poewe W; SP716 Study Group; SP715 Study Group. Rotigotine transdermal system for long-term treatment of patients with advanced Parkinson's disease: results of two open-label extension studies, CLEOPATRA-PD and PREFER. J Neural Transm. 2013;120(7):1069-1081.

14. Ray Chaudhuri K, Martinez-Martin P, Antonini A, et al. Rotigotine and specific non-motor symptoms of Parkinson's disease: post hoc analysis of RECOVER. Parkinsonism Relat Disord. 2013;19(7):660-665.

15. Zhou CQ, Li SS, Chen ZM, Li FQ, Lei P, Peng GG. Rotigotine transdermal patch in Parkinson's disease: a systematic review and meta-analysis. PLoS One. 2013;8(7):e69738.

16. Wenning GK, Ben-Shlomo Y, Magalhães M, Daniel SE, Quinn NP Clinicopathological study of 35 cases of multiple system atrophy. J Neurol Neurosurg Psychiatry. 1995;58(2):160-166.

17. Wenning GK, Tison F, Ben Shlomo Y, Daniel SE, Quinn NP. Multiple system atrophy: a review of 203 pathologically proven cases. Mov Disord. 1997;12(2):133-147.

18. Wenning GK, Scherfler C, Granata R, et al. Time course of symptomatic orthostatic hypotension and urinary incontinence in patients with postmortem confirmed parkinsonian syndromes: a clinicopathological study. J Neurol Neurosurg Psychiatry. 1999;67(5):620-623.

19. Wenning GK, Stefanova N, Jellinger KA, Poewe W, Schlossmacher MG Multiple system atrophy: a primary oligodendrogliopathy. Ann Neurol. 2008;64(3):239-246.

20. Litvan I, Mangone CA, McKee A, et al. Natural history of progressive supranuclear palsy (Steele-Richardson-Olszewski syndrome) and clinical predictors of survival: a clinicopathological study. J Neurol Neurosurg Psychiatry. 1996;60(6):615-620.

21. Litvan I, Agid Y, Calne D, et al. Clinical research criteria for the diagnosis of progressive supranuclear palsy (Steele-Richardson-Olszewski syndrome): report of the NINDS-SPSP international workshop. Neurology. 1996;47(1):1-9.

22. Williams DR, Lees AJ. Progressive supranuclear palsy: clinicopathological concepts and diagnostic challenges. Lancet Neurol. 2009;8(3):270-279.

23. Ling H, O'Sullivan SS, Holton JL, et al. Does corticobasal degeneration exist? A clinicopathological re-evaluation. Brain. 2010;133(Pt 7):2045-2057.

24. Liscic RM, Srulijes K, Gröger A, Maetzler W, Berg D. Differentiation of progressive supranuclear palsy: clinical, imaging and laboratory tools. Acta Neurol Scand. 2013;127(5):362-370.

25. Larsen JP, Dupont E, Tandberg E. The clinical diagnosis of Parkinson's disease. Proposal of diagnostic subgroups classified at different levels of confidence. Acta Neurol Scand. 1994;89(4):242-251.

26. Aarsland D, Litvan I, Salmon D, Galasko D, Wentzel-Larsen T, Larsen JP Performance on the dementia rating scale in Parkinson's disease with dementia and dementia with Lewy bodies: comparison with progressive supranuclear palsy and Alzheimer's disease. J Neurol Neurosurg Psychiatry. 2003;74(9):1215-1220.

27. Berardelli A, Wenning GK, Antonini A, et al. EFNS/MDS-ES/ENS [corrected] recommendations for the diagnosis of Parkinson's disease. Eur J Neurol. 2013;20(1):16-34. Erratum in: Eur J Neurol. 2013; 20(2):406.
28. Stamelou M, Hoeglinger GU. Atypical parkinsonism: an update. Curr Opin Neurol. 2013;26(4):401-405.

29. McKeith IG, Galasko D, Kosaka K, et al. Consensus guidelines for the clinical diagnosis of dementia with Lewy bodies (DLB): report of the consortium on DLB international workshop. Neurology. 1996;47(5):1113-1124.

30. McKeith IG, Dickson DW, Lowe J, et al; Consortium on DLB. Diagnosis and management of dementia with Lewy bodies: third report of the DLB Consortium. Neurology. 2005;65(12):1863-1872.

31. Fahn S, Elton RL; UPDRS Program Members. Unified Parkinson's disease rating scale. In: Fahn S, Marsden CD, Goldstein M, Calne DB, editors. Recent Developments in Parkinson's Disease. Vol. 2. Florham Park, NJ: Macmillan Healthcare Information; 1987:153-163, 293-304.

32. Cummings JL, Mega M, Gray K, Rosenberg-Thomson S, Carusi DA, Gornbein J. The Neuropsychiatric Inventory: comprehensive assessment of psychopathology in dementia. Neurology. 1994;44(12): 2308-2314.

33. Folstein MF, Folstein SE, McHugh PR. "Mini-mental state". A practical method for grading the cognitive state of patients for the clinician. J Psychiatr Res. 1975;12(3):189-98.

34. Poewe W, Barone P, Gliadi N, et al. A randomized, placebo-controlled clinical trial to assess the effects of rasagiline in patients with multiple system atrophy of the parkinsonian subtype. Mov Disord. 2012;27(Suppl 1): abstr 1182.

35. Novak P, Williams A, Ravin P, Zurkiya O, Abduljalil A, Novak V. Treatment of multiple system atrophy using intravenous immunoglobulin. BMC Neurol. 2012;12:131.

36. Lee PH, Lee JE, Kim HS, et al. A randomized trial of mesenchymal stem cells in multiple system atrophy. Ann Neurol. 2012;72(1):32-40.

37. Höglinger GU, Huppertz HJ, Wagenpfeil S, et al; TAUROS MRI Investigators. Tideglusib reduces progression of brain atrophy in progressive supranuclear palsy in a randomized trial. Mov Disord. 2014;29(4): 479-487.

38. Stamelou M, de Silva R, Arias-Carrión O, et al. Rational therapeutic approaches to progressive supranuclear palsy. Brain. 2010;133(Pt 6): $1578-1590$

39. Lieberman A, Olanow CW, Sethi K, et al. A multicenter trial of ropinirole as adjunct treatment for Parkinson's disease. Ropinirole Study Group. Neurology. 1998;51(4):1057-1062.

40. Lieberman A, Ranhosky A, Korts D. Clinical evaluation of pramipexole in advanced Parkinson's disease: results of a double-blind, placebocontrolled, parallel-group study. Neurology. 1997;49(1):162-168.

41. LeWitt PA, Lyons KE, Pahwa R; SP 650 Study Group. Advanced Parkinson disease treated with rotigotine transdermal system: PREFER study. Neurology. 2007;68(16):1262-1267.

42. Georgiev D, Danieli A, Ocepek L, et al. Othello syndrome in patients with Parkinson's disease. Psychiatr Danub. 2010;22(1):94-98.

43. Scheller D, Ullmer C, Berkels R, Gwarek M, Lübbert H. The in vitro receptor profile of rotigotine: a new agent for the treatment of Parkinson's disease. Naunyn Schmiedebergs Arch Pharmacol. 2009;379(1):73-86.

44. Scheller D, Stichel-Gunkel C, Lübbert H, et al. Neuroprotective effects of rotigotine in the acute MPTP-lesioned mouse model of Parkinson's disease. Neurosci Lett. 2008;432(1):30-4.

45. Zanettini R, Antonini A, Gatto G, Gentile R, Tesei S, Pezzoli G. Valvular heart disease and the use of dopamine agonists for Parkinson's disease. N Engl J Med. 2007;356(1):39-46.
Neuropsychiatric Disease and Treatment

\section{Publish your work in this journal}

Neuropsychiatric Disease and Treatment is an international, peerreviewed journal of clinical therapeutics and pharmacology focusing on concise rapid reporting of clinical or pre-clinical studies on a range of neuropsychiatric and neurological disorders. This journa is indexed on PubMed Central, the 'PsycINFO' database and CAS,

\section{Dovepress}

and is the official journal of The International Neuropsychiatric Association (INA). The manuscript management system is completely online and includes a very quick and fair peer-review system, which is all easy to use. Visit http://www.dovepress.com/testimonials.php to read real quotes from published authors. 